R. Asero ${ }^{1}$, A. Aruanno 2 , M. Brescianin ${ }^{3}$, I. Brusca ${ }^{4}$, M. Carollo 5 , L. Cecchi ${ }^{6}$, G. Cortellini ${ }^{7}$, G. Deleonardi ${ }^{8}$, A. Farsi ${ }^{6}$, E. Ferrarini ${ }^{9}$, G. Gabrielli ${ }^{10}$, A. Ingrassia ${ }^{11}$, M. Mauro $^{12}$, F. Murzilli ${ }^{13}$, E. Nucera ${ }^{2}$, R. Onida ${ }^{4}$, E. A. Pastorello ${ }^{14}$, E. Pinter ${ }^{15}$, A. Rizzi ${ }^{2}$, M. Russello ${ }^{12}$, C. SaCerdoti ${ }^{16}$, E. Scala ${ }^{17}$, G. Scala ${ }^{18}$, D. Villalta ${ }^{19}$, S. Zampogna ${ }^{20}$, S. Amato ${ }^{21}$, G. Mistrello ${ }^{21}$

\title{
Evaluation of two commercial peach extracts for skin prick testing in the diagnosis of hypersensitivity to lipid transfer protein. A multicenter study
}

\author{
${ }^{1}$ Allergology Clinic, Clinica San Carlo, Paderno Dugnano, Milan, Italy \\ ${ }^{2}$ Fondazione Policlinico Universitario A. Gemelli-IRCCS, Rome, Italy \\ ${ }^{3}$ Allergology Clinic, Asl RM 4, S. Paolo Civitavecchia Hospital, Civitavecchia, Italy \\ ${ }^{4}$ Clinical Pathology U.O.C., Buccheri La Ferla F.B.F. Hospital, Palermo, Italy \\ ${ }^{5}$ Pathology and Clinical Biochemistry, Magna Graecia University, Catanzaro, Italy \\ ${ }^{6}$ Allergology and Immunology SOS, Prato-Azienda USL Toscana Centro, Prato, Italy \\ ${ }^{7}$ Romagna Allergy Unit, Internist Department of Rimini, Rimini, Italy \\ ${ }^{8}$ Laboratorio Unico Metropolitano, AUSL Bologna, Bologna, Italy \\ ${ }^{9}$ Allergology OUS, Umberto I Hospital, Siracusa, Italy \\ ${ }^{10}$ School of Specialization in Allergy and Clinical Immunology, Alma Mater Studiorum, Bologna, Italy \\ ${ }^{11}$ Allergology UO, District of Marsala, ASP 9, Trapani, Italy \\ ${ }^{12}$ Allergology UO, ASST Lariana, Como, Italy \\ ${ }^{13}$ Allergology U.O.S.D., S.S. Filippo e Nicola Hospital, Avezzano, Italy \\ ${ }^{14}$ Struttura Complessa di Allergologia e Immunologia, ASST GOM, Niguarda, Milan, Italy \\ ${ }^{15}$ Clinic Immunology U.O.C., Policlinico Umberto Primo, Rome, Italy \\ ${ }^{16}$ Private Practice, Naples, Italy \\ ${ }^{17}$ Istituto Dermopatico dell'Immacolata, Rome, Italy \\ ${ }^{18}$ Allergology UOSD, Loreto Crispi, ASL Napoli 1 Centro, Naples, Italy \\ ${ }^{19}$ Immunology and Allergology Unit, Santa Maria degli Angeli Hospital, Pordenone, Italy \\ ${ }^{20}$ Pediatric First Aid, Pugliese Ciaccio Hospital, Catanzaro, Italy \\ ${ }^{21}$ Lofarma SpA, R \& D, Milan, Italy
}

\section{KEY WORDS}

Food allergy; Peach allergy; lipid transfer protein; SPT; diagnosis.

\author{
Corresponding author \\ Riccardo Asero \\ Allergology Clinic \\ Clinica San Carlo \\ via Ospedale 21 \\ 20037 Paderno Dugnano, Milan, Italy \\ E-mail: r.asero@libero.it \\ Doi \\ 10.23822/EurAnnACI.1764-1489.144
}

( 2021 Associazione Allergologi Immunologi Italiani Territoriali e Ospedalieri - AAllTO. Published by EDRA SpA. All rights reserved.

\begin{abstract}
Summary
The clinical usefulness of two commercial peach extracts for SPT (by Lofarma $S p A$ and ALK-Abello, respectively) was compared in a multicenter study carried out in Italy. Peach allergic patients were tested with the two extracts in parallel and underwent the detection of IgE specific for all three peach allergens currently available (Pru p1, Pru p3, and Pru p4, respectively). The two extracts were almost identical in terms of sensitivity and specificity, being able to detect virtually all patients sensitized to stable peach allergens (lipid transfer protein (LTP) and, presumably, peamaclein) but scoring negative in patients exclusively sensitive to labile allergens (either PR-10 andlor profilin). Thus, the two extracts represent an excellent tool to carry out a preliminary component-resolved diagnosis of peach allergy at the first patient visit.
\end{abstract}




\section{Introduction}

In the clinical routine practice, the diagnosis of allergic diseases is based on a) convincing clinical history, b) demonstration of hypersensitivity to one or more allergen sources, and c) provocation tests (in specific cases only). The detection of IgE-mediated hypersensitivity is traditionally (and still in most cases) accomplished by skin prick tests (SPT) with commercial extracts of different allergen sources. The in-vitro detection of IgE specific for either allergen sources or single allergen components is generally reserved to cases in which skin testing is not feasible (e.g., during antihistamine treatment) or in the presence of skin reactivity to a large number of different sources due to sensitization to cross-reacting pan-allergens. The EU decree 2001/83/EG establishes that solutions for skin tests are legally assimilable to drugs and must be licensed by individual national regulatory agencies based on strict quality control. The elevated costs of regulatory requirements are leading to the gradual withdrawal of the less commonly employed preparations by the producing companies and, obviously, the loss of diagnostic in vivo extracts has involved mainly food allergen extracts. The lack of commercial SPT food extracts can be replaced by SPT with fresh material, which is unfortunately often not feasible, or by in vitro tests with an undeniably significant increase in costs for both the patients and the NHS, and of more time wasted by patients and doctors. Thus, the availability of newly licensed food extracts for SPT is welcome.

Recently, the Italian regulatory agency, AIFA (Agenzia Italiana del Farmaco) admitted to the licensing process a commercial peach extract for SPT produced by Lofarma, Milan, Italy. The availability of commercial peach extracts for SPT in a Mediterranean country like Italy is of the utmost importance, as they most likely lack labile allergens (i.e., Pru p1, the PR-10 allergen homologous to the major birch pollen allergen, Bet v1, and Pru p4, the peach profilin) which are lost during the production procedures while retaining stable allergens like Pru p3 (the nonspecific Lipid Transfer Protein, LTP) and Pru p7 (the gibberellin-regulated protein, also known as peamaclein) (1-3). Therefore, SPT with these extracts represents an essential means to carry out a prompt, first-level component-resolved diagnosis at the bedside, getting immediately very important information from a clinical and prognostic point of view $(1,4)$. Notably, LTP is by far the most frequent primary food allergy in Italy (5), and the main cause of food-induced anaphylactic reactions (6). So far, clinical studies dealing with food allergy induced by Rosaceae or with allergy to LTP have been carried out using a commercial peach extract enriched with Pru p3 (declaring a concentration of $30 \mathrm{mg} / \mathrm{ml}$ Pru p3) by ALK-Abellò, Madrid, Spain (7-10). In the present multicenter study, we analyzed another commercial peach extract produced by Lofarma, Milan, Italy comparing its clinical usefulness with that of the mentioned ALK-Abellò extract in a large population of peach-allergic patients sensitized to different peach allergens.

\section{Methods}

\section{Commercial peach peel SPT extract}

The commercial peach (pulp + peel) extract for SPT was prepared by Lofarma S.p.A. laboratories, Milan Italy. The final concentration of Pru p3 in this extract is adjusted to $50 \mu \mathrm{g} / \mathrm{ml}$ before commercialization.

The peach extract was distributed to the allergy centers scattered throughout the country who participated in the study.

\section{Patients and skin tests}

Patients with a convincing clinical history of peach allergy (i.e., clear-cut oral allergy syndrome, urticaria/angioedema, or anaphylaxis following by less than 2 hours the ingestion of peach) were enrolled in the study. After signing an informed consent, patients underwent SPT with both Lofarma and ALK-Abellò peach extracts. Skin prick tests were carried out following established methods using disposable commercial $1 \mathrm{~mm}$ tip lancets (the single centers were left free to use their usual commercial lancets). The two commercial peach extracts were tested in parallel. Histamine $10 \mathrm{mg} / \mathrm{ml}$ and saline were used as positive and negative controls, respectively. Readings were taken at $15 \mathrm{~min}$, and the mean diameter of the wheal was measured. Wheals exceeding $3 \mathrm{~mm}$ were considered positive.

\section{In vitro tests}

IgE to Pru p1 (or Bet v1), Pru p3, and Pru p4 (or Phl p12) were measured by ImmunoCAP. Levels exceeding $0.1 \mathrm{kU} / \mathrm{L}$ were considered positive. Patients scoring positive for Pru p1/Bet v1 and/or Pru p4/Phl p12 in the absence of detectable reactivity to Pru p3 were considered as possibly sensitized uniquely to labile peach allergens.

\section{Results}

Two-hundred forty-four peach-allergic patients (M/F 155/89; mean age 30.7 years, range 5-68 years) were finally enrolled. Of these, 220 scored positive on SPT with both $(n=216)$ or one ( $n$ = 4) peach extract showing an almost perfect agreement $(99.5 \%$; $\mathrm{p}<0.0001$ ) between the two tests. In the four patients showing a discrepancy between the two SPT extracts, Lofarma extract scored negative in 3 cases and ALK-Abellò extract in 1 case. These 4 patients showed Pru p3 IgE levels ranging from 0.45 to $3.2 \mathrm{kU} / \mathrm{L}$. Although this did not cause appreciable differences in sensitivity between the two study extracts, several centers reported that the size of the wheals produced by the ALK-Abello extract frequently slightly exceeded those induced by the Lofarma extract.

Patients scoring positive for one or both peach SPT extracts showed IgE to Pru p3 in 198/202 (98\%) cases; 4 patients did not show any IgE reactivity to PR-10, profilin, or Pru p3 on ImmmunoCAP analysis despite being strongly sensitized to peach (both extracts positive 
on SPT) and having clinical history of systemic reactions. Therefore, they were classified as being sensitized to a stable allergen other than the lipid transfer protein, possibly peamaclein or another yet not identified peach allergen (10). IgE to Pru p3 was not measured in 18 patients (all scoring positive on SPT with both extracts).

Twenty-four patients were sensitized exclusively to labile allergens (i.e., they showed $\operatorname{IgE}$ reactivity to either PR-10 and/or Profilin in the absence of $\mathrm{IgE}$ reactivity to Pru p3, the peach LTP). All of them scored negative on skin tests with both peach extracts. The results of the study are summarized in table $\mathbf{I}$.

Table I - Skin and serological tests carried out in 244 peach-allergic patients.

\begin{tabular}{lcc}
\hline ImmunoCAP & Lofarma SPT $^{+}$ & ALK SPT $^{+}$ \\
\hline Pru p3 $^{+}(\mathrm{n}=198)$ & 195 & 197 \\
\hline Pru p1 or 4+/Pru p $(\mathrm{n}=24)$ & 0 & 0 \\
\hline Pru p1, 3, 4 $^{-}(\mathrm{n}=4)$ & 4 & 4 \\
\hline Not tested $(\mathrm{n}=18)$ & 18 & 18 \\
\hline
\end{tabular}

\section{Discussion}

Clinical allergists strongly need instruments able to identify already at the first visit whether the patient presenting for a consultancy has an allergy or not. The license regulations decreed by the EU and adopted by the National regulatory agencies pose a serious risk to make this task extremely difficult or even impossible with a consequent exponential increase of costs for the patients and the community and of time expenditures by both doctors and patients. Therefore, the availability of diagnostic instruments for in vivo diagnosis of food allergy is welcome. In the present study, we compared the clinical usefulness and performance of two commercial peach extracts, one by Lofarma SpA and the other by ALKAbellò in a large group of peach-allergic patients. The two tests showed a nearly identical efficiency: both scored negative in patients sensitized uniquely to labile peach allergens and positive in most patients sensitized to stable peach allergens. This feature of the two preparations allows to carry out a component-resolved diagnosis of peach allergy at the first clinical visit, identifying patients who are at a greater risk of systemic reactions. Interestingly, both extracts were able to identify four patients sensitized to stable peach allergens other than LTP.

\section{Conclusions}

The study confirmed that peach labile allergens are lost during the extraction procedures and that SPT with fresh material are needed to detect patients sensitized to such allergens in the clinical practice. The main limitation of this study is the lack of a correlation analysis between the level of IgE specific for LTP and the size (either area or mean diameter) of the wheal produced by the two extracts on SPT. However, in view of the high variability of skin responses between patients showing equal levels of specific $\mathrm{IgE}$, it seems unlikely that such analysis would have added further useful information to the current findings. Therefore, it is possible to conclude that both peach extracts studied are clinically useful to detect/exclude sensitization to stable peach allergens.

\section{Conflict of interests}

The authors declare that they have no conflict of interests.

\section{References}

1. Asero R. Detection and clinical characterization of patients with oral allergy syndrome caused by stable allergens in Rosaceae and nuts. Ann Allergy Asthma Immunol 1999;83(5):377-83.

2. Asero R, Mistrello G, Roncarolo D, Casarini M, Falagiani P. Allergy to nonspecific lipid transfer proteins in Rosaceae: a comparative study of different in vivo diagnostic methods. Ann Allergy Asthma Immunol 2001;87:68-71.

3. Tuppo L, Alessandri C, Pomponi D, et al. Peamaclein-a new peach allergenic protein: similarities, differences and misleading features compared to Pru p3. Clin Exp Allergy 2013;43:128-40.

4. Asero R, Jimeno L, Barber D. Component-resolved diagnosis of plant food allergy by SPT. Eur Ann Allergy Clin Immunol 2008;40:115-21.

5. Asero R, Antonicelli L, Arena A, et al. EpidemAAITO: features of food allergy in Italian adults attending allergy clinics: a multi-centre study. Clin Exp Allergy 2009;39:547-55.

6. Asero R, Antonicelli L, Arena A, et al. Causes of food-induced anaphylaxis in Italian adults: a multi-centre study. Int Arch Allergy Immunol 2009;150:271-7.

7. Goikoetxea MJ, Berroa F, Cabrera-Freitag P, et al. Do Skin Prick Test and In Vitro Techniques Diagnose Sensitization to Peach Lipid Transfer Protein and Profilin Equally Well in Allergy to Plant Food and Pollen? J Investig Allergol Clin Immunol 2015;25:283-7.

8. Barber D, de la Torre F, Lombardero M, et al. Component-resolved diagnosis of pollen allergy based on skin testing with profilin, polcalcin and lipid transfer protein pan-allergens. Clin Exp Allergy 2009;39:1764-73.

9. Asero R. Lipid transfer protein cross-reactivity assessed in vivo and in vitro in the office: pros and cons. J Investig Allergol Clin Immunol 2011;21:129-36.

10. Asero R, Abbadessa S, Aruanno A, et al. Detection of gibberellin-regulated protein (peamaclein) among Italian cypress pollen-sensitized patients. J Investig Allergol Clin Immunol 2020. Ahead of Print. 